\title{
Analysis of Heavy Metal Pollution Based on Two-Dimensional Diffusion Model
}

\author{
Yunhui Zeng \\ College of Intelligent Science and Engineering, Jinan University, No. 206, Qianshan Road, Xiangzhou District, Zhuhai City, Guangdong \\ Province, China \\ Wenhao Li \\ College of Intelligent Science and Engineering, Jinan University, No. 206, Qianshan Road, Xiangzhou District, Zhuhai City, Guangdong \\ Province, China \\ Hongfei Guo* \\ College of Internet of Things and Logistics Engineering, Jinan University, No. 206, Qianshan Road, Xiangzhou District, Zhuhai City, \\ Guangdong Province, China \\ Yilin Chen \\ College of Intelligent Science and Engineering, Jinan University, No. 206, Qianshan Road, Xiangzhou District, Zhuhai City, Guangdong \\ Province, China

\section{Xiaoqing Jiang} \\ School of translation studies, Jinan University, No. 206, Qianshan Road, Xiangzhou District, Zhuhai City, Guangdong Province, China

\section{Bingjie Yu} \\ College of Intelligent Science and Engineering, Jinan University, No. 206, Qianshan Road, Xiangzhou District, Zhuhai City, Guangdong \\ Province, China
}

\begin{abstract}
This paper takes the propagation characteristics of heavy metals and the judgment of the location of pollution sources as the research objects, aiming to analyze the propagation characteristics of different heavy metals. Firstly, the Gaussian diffusion model is conducted to establish the propagation characteristics model of heavy metal pollutants in the atmosphere. Then, based on the law of conservation of mass and the law of two-dimensional diffusion, the two-dimensional diffusion model is adopted to establish the propagation characteristics model of heavy metals in soil moisture. According to these two models, the nonlinear differential equations are established respectively, revealing that the characteristics of the two propagation ways are related to space, time, diffusion coefficients, and other factors. Then, in the light of the propagation characteristics of different heavy metals, the least square method is applied to reduce the data calculation error and obtain the specific location of the pollution source. Finally, through establishing the three-dimensional diffusion model of heavy metal diffusion by introducing artificial control, speed and angle of prevailing wind direction, and other factors, the model is further optimized. The establishment of this model provides an important theoretical basis and guiding significance for the future study of heavy metal pollutants.
\end{abstract}

Keywords: Heavy metal pollution; Gaussian diffusion model; Nonlinear differential; Least square method; Three-dimensional diffusion model.

\section{(9) (i) CC BY: Creative Commons Attribution License 4.0}

\section{Introduction}

Soil heavy metal pollution refers to the phenomenon of high heavy metal content caused by excessive deposition of trace metal elements in soil due to human activities. With the rapid development of urban economy and the continuous increase of urban population, the impact of human activities on urban environmental quality is increasingly prominent. Due to the hysteresis, concealment and long-term nature of soil heavy metal pollution, the pollution and ecological problems caused often fail to attract enough attention. The focus of people's attention gradually moves to verify geological environment anomaly in urban soil as well as how to carry out urban environmental quality assessment and study the evolution model of urban geological environment under the influence of human activities by applying massive data obtained from verification [1]. Out of the current heavy metal pollution researches which mainly focus on analyzing the ways and sources of pollution, there are few researches target at analyzing the propagation characteristics of heavy metal pollution and determining the specific location of pollution sources at home and abroad. Therefore, researchers aimed at determining the specific location of pollution sources are of certain necessity. The equal-standard pollution load method proposed by Zhao Jinjin [2]obtained the location of pollution sources by simply listing the concentration of different element pollutants at different locations (Zhao Wei) and making intercomparison, deviating from scientific results. Wang Ying, Jiang Xiaodong and other people proposed to use the convection-diffusion model in the propagation medium to describe the migration process of heavy metal pollutants in soil [3] only considering the propagation of heavy metals in a certain direction. Zhang 
Ganlin and Zhu Yongguan and other people adopted Gaussian diffusion model to study the determination of gas pollution sources [4], which was not completely applicable to the determination of heavy metal pollution sources in soil. In general, the analysis of the propagation of heavy metal pollutants in the academic circle generally has the following problems: The result analysis lacks rigorously scientific calculation; fail to consider the correlation between heavy metals; the propagation mode of heavy metals is not comprehensive; the method adopted is too idealized to be practical.

Aiming at the propagation characteristics of heavy metal pollutants and the location of pollution sources, the Gaussian smoke mass diffusion model is conducted in this paper to establish the propagation characteristics model of heavy metal pollutants under the action of the atmosphere and the propagation characteristics model under the action of soil moisture. The propagation mode of heavy metal pollution is probed into more comprehensively, making the model more practical. Then, based on the law of conservation of mass and the law of two-dimensional diffusion, the two-dimensional diffusion model is adopted to establish the propagation model of heavy metals in soil moisture, and nonlinear differential equations are established respectively for the two cases. Finally, through establishing the threedimensional diffusion model of heavy metal diffusion by introducing artificial control, speed and angle of prevailing wind direction, and other factors, the model is further optimized and thus become more comprehensive and scientific.

\section{Measurements}

\subsection{Data Acquisition and Assumptions}

The original data of this paper is from China Society for Industrial and Applied Mathematics, 2011, including the concentration of eight major heavy metal elements at the sampling point and their background values. For facilitating analysis and understanding, we make the following assumptions about the model:

(1) The data given in the title is true and authentic;

(2) No degradation of heavy metals;

(3) Assuming the same probability of airflow occurring in all directions, that is, the propagation characteristic of atmosphere is spherical diffusion;

(4) Ignore the influence of gravity on heavy metal ions.

\subsection{Model Establishment and Solution}

\subsubsection{Model for the Propagation of Heavy Metals in the Atmosphere [5]}

The propagation of heavy metals in the air is characterized by spread in all directions. The atmospheric airflow is constantly changing, which is random. Therefore, it's assumed that the probability of airflow in all directions is the same, so the propagation characteristics can be considered as spherical diffusion to make the Gaussian diffusion model [6] applicable. Thus the pollution resource is recorded as $\left(x_{0}, y_{0}, z_{0}\right)$, the concentration of pollution area as $C(x, y, z, 0)$ and the concentration of the heavy metal at any point in the infinite space from the pollution source ${ }^{\mathrm{t}}$ as $C(x, y, z, \mathrm{t})$. The flow rate through the unit normal area within the unit distance is proportional to the concentration gradient. Therefore,

$$
\bar{q}=-k_{\text {air }} \times \operatorname{gradC}
$$

$k_{\text {air }}$ is the diffusion coefficient of a certain heavy metal in the air, grad indicates the gradient, and the symbol indicates that it diffuses from the high concentration to the low concentration. Then the flow through the time interval $[t, t+\mathrm{D} t]_{\text {is: }}$

$$
M_{1}=\grave{\mathrm{O}}_{t}^{t+\mathrm{D} t} \text { òò }_{s} q p d s d t
$$

$\mathrm{W}_{\text {stands for Inter-space domain, }} V$ for the volume of $\mathrm{W}, N$ for the surface surrounding of $\mathrm{W}$ and $p$ for the outer normal vector of $N$. The increase of heavy metal ions in $\mathrm{W}$ is:

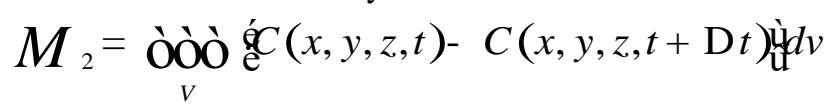

According to the law of conservation of mass :

$$
\begin{aligned}
& M_{2}=M_{1} \\
& \text { And the Gauss formula of the surface area: } \\
& \text { ÒÒ } q p d s=\underset{S}{=} \text { Òò } d i v \text { q } d v
\end{aligned}
$$

$d i v$ is the divergence mark. Based on the above formula, the integral mean value theorem is conducted to be: 


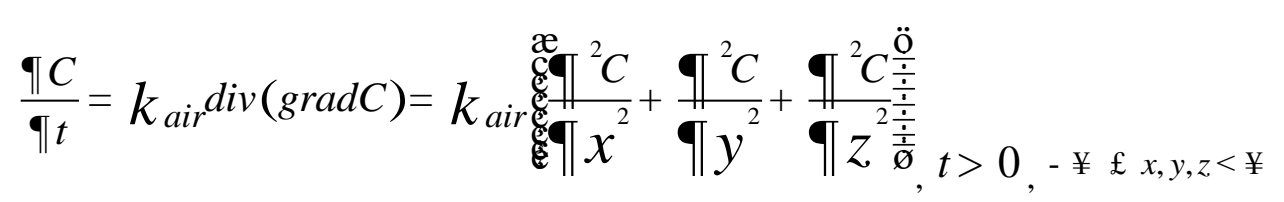

The heavy metal diffusion is regarded as the instantaneous and equal release of heavy metal pollutants to all directions from a certain pollution source. The initial condition is the point source function acting on the pollution source coordinates, which can be recorded as:

$$
y\left(x_{0}, y_{0}, z_{0}\right)=M s\left(x_{0}, y_{0}, z_{0}\right)
$$

$M$ represents the total amount of heavy metal release, the initial concentration of heavy metals at time 0 ; $s\left(x_{0}, y_{0}, z_{0}\right)$

$$
C(x, y, z, t)=\frac{M}{\left(4 p k_{a i r} t\right)^{3 / 2}} e^{-\frac{\left(x-x_{0}\right)^{2}+\left(y-y_{0}\right)^{2}+(z-z 0)^{2}}{{ }^{4} k_{a i t} t}}
$$

The result illustrates that the isosurface of the diffusion concentration $\mathrm{Y}$ of heavy metals in the atmosphere is a spherical surface at any time $t$, and the value of the concentration $R$ decreases continuously as the distance $\mathrm{Y}$ increases away from the pollution source.

\subsubsection{Metal Leachate Diffusion Model in the Soil}

The presence of moisture in the soil will lead to heavy metals diffusion in the form of ions. Heavy metals mainly diffuse from high concentration to low concentration in the surface layer, which mainly affected by the concentration of heavy metals. Therefore, a two-dimensional propagation model is established based on the horizontal diffusion, regardless of the influence of altitude. [7]:

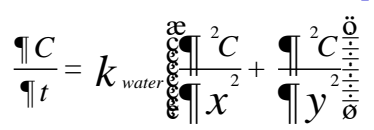

$C$ stands for the concentration of heavy metals, and $k_{\text {water }}$ for the diffusion coefficient of certain heavy metal in the soil.

With the pollution source coordinate $\left(x_{0}, y_{0}\right)$, the initial concentration $M$, and solution of the partial differential equation in the two-dimensional propagation model, the expression of the concentration of heavy metal pollutants at the moment can be obtained:

$$
C(x, y, t)=\frac{M}{8(p t)^{3 / 2} k_{\text {water }}} e^{-\frac{\left(x-x_{0}\right)^{2}+\left(y-y_{0}\right)^{2}}{{ }^{4 t} k_{\text {water }}}}
$$

$\left(x_{0}, y_{0}\right)$ stands for the pollution source coordinate and $M$ for the initial concentration.

\subsubsection{Analysis of Propagation Characteristics}

The model analysis of the two propagation characteristics reveals that the farther the heavy metal is from the pollution source, the lower the concentration is. Therefore, the point with the highest concentration of heavy metals can be inferred as the source of pollution.

Elements with larger relative molecular mass sink faster and are difficult to move farther through the atmosphere, thus the heavy metals are divided into groups by their relative molecular masses. The smaller relative molecular masses of $\mathrm{Cu}, \mathrm{Ni}, \mathrm{As}, \mathrm{Zn}$ and $\mathrm{Cr}$ are mainly diffused by atmosphere while $\mathrm{Pb}, \mathrm{Hg}$ and $\mathrm{Cd}$ that have larger relative molecular masses are mainly diffused by soil leachate.

2.2.4 Determination of Pollution Sources

Taking the $A s$ element as an example, some points near the highest concentration point of $A s$ are selected to calculate the location of the main pollution source, trying to comply with the assumption of the model. Selection criteria: measured concentration values: $C>5$; abscissa: $16000<x<20000$; ordinate: $8000<y<20000$. Finally, 10 points that meet the requirements are selected. The results are displayed in Table 1: 
Table-1. Ten points that meet the requirements

\begin{tabular}{l|l|l|l|l|l|l|l|l|l|l}
\hline $\mathrm{C}$ & 8.5 & 10.99 & 6.35 & 30.13 & 6.98 & 5.41 & 5.83 & 6.14 & 6.69 & 5.41 \\
\hline $\mathrm{x}$ & 17087 & 18738 & 17814 & 18134 & 19767 & 16301 & 17904 & 19007 & 19072 & 16289 \\
\hline $\mathrm{y}$ & 11933 & 10921 & 10707 & 10046 & 8810 & 8299 & 8287 & 11488 & 8519 & 10072 \\
\hline
\end{tabular}

Approximately, the value of $z$ and ${ }^{t}$ of these points is the same. In order to reduce the data error and match the actual situation, linear regression [8] that based on the principle of least squares method [9] is applied to obtain the value of $(x, y)$, putting a maximum value of $C$. The result is $(x, y)=(18102,10066)$. The main pollution source locations of the remaining seven elements are worked out by the sam e method, which are showed in Table 2 .

Table-2. The main pollution source locations of the eight elements

\begin{tabular}{l|l|l|l}
\hline elements & $\mathbf{x}$ & $\mathbf{y}$ & concentration \\
\hline $\mathrm{As}$ & 18102 & 10066 & 30.59 \\
\hline $\mathrm{Cd}$ & 21449 & 11386 & 1621.21 \\
\hline $\mathrm{Cu}$ & 2383 & 3692 & 2528.48 \\
\hline $\mathrm{Cr}$ & 3299 & 6018 & 920.84 \\
\hline $\mathrm{Hg}$ & 2708 & 2295 & 16000 \\
\hline $\mathrm{Ni}$ & 3305 & 6022 & 144.2 \\
\hline $\mathrm{Pb}$ & 4778 & 4901 & 475.33 \\
\hline $\mathrm{Zn}$ & 13797 & 9621 & 3760.82 \\
\hline
\end{tabular}

\subsubsection{The Introduction of Three-Dimensional Diffusion Model with Human Governance}

The locations of the pollution sources can be affected by natural factors in the actual environment, the paper adds considerations of human factors into the influence on the original three-dimensional diffusion model [10]. The intensity of the artificial control can be illustrated as a reduction factor of pollution concentration (but inviting investment to build factories can be illustrated as an increase coefficient of pollution concentration). The artificial pollution control can be assumed as a constant $G$ within the range of $0 \leq \mathrm{G} \leq 1$, then its three-dimensional diffusion model that match the actual situation is built:

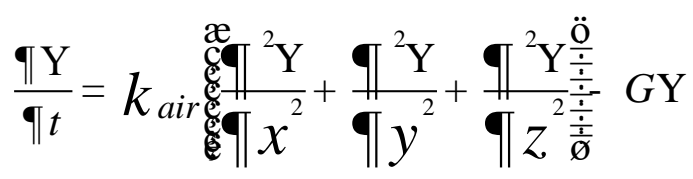

$(t \geq 0,-\infty \leq \mathrm{x} \leq \infty,-\infty \leq y \leq \infty), k_{\text {air }}$ represents the diffusion coefficient of heavy metals in the air, and

$G$ represents the artificial pollution control.

The heavy metal diffusion is regarded as the instantaneous and equal release of heavy metal pollutants to all directions from a certain pollution source. The initial condition is the point source function acting on the pollution source coordinate $\left(x_{0}, y_{0}, z_{0}\right)$, which can be recorded as:

$$
y\left(x_{0}, y_{0}, z_{0}\right)=\operatorname{Ms}\left(x_{0}, y_{0}, z_{0}\right)
$$

$M$ indicates the total amount of heavy metal release, the initial concentration of heavy metals at time 0 ; $s\left(x_{0}, y_{0}, z_{0}\right)$ is the point source function of diffusion intensity in each unit. The solution of equation (11) that satisfied equation (12) is:

$$
\mathrm{Y}(x, y, z, t)=\frac{M}{\left(4 p k_{\text {air }} t\right)^{3 / 2}} e^{-\frac{\left(x-x_{0}\right)^{2}+\left(y-y_{0}\right)^{2}+\left(z-z_{0}\right)^{2}}{{ }^{4} k_{\text {air }}{ }^{2}}}(G \mathrm{t})
$$

With the above results, the artificial pollution control $\mathrm{G}$, and other values such as $k$ air , the concentration of each metal at a given position is worked out.

The calculation results show that a more effective artificial pollution control will bring a lighter pollution in the same area and a smaller polluted area for the same pollution source. This is because the artificial pollution control has a great impact on changing the pollution concentration. The more human governs, the better the effect is, which is in line with the actual situation.

The above analysis tells that a strong control on the pollution sources and the heavily polluted areas will gain a more significant effect when conducting heavy metal pollution control and treatment. 


\subsubsection{The Introduction of a Three-Dimensional Diffusion Model with Constant Wind Direction}

Apart from the introduction of the three-dimensional diffusion model of artificial control in the previous section, the paper also considers the actual influence of the prevailing wind direction on the three-dimensional diffusion model so that the results obtained by the model are closer to reality. The so-called prevailing wind direction is the main wind direction of a wind in the city (if the conditions are enough, it can be defined a function of wind direction over time, converting it into a variable wind direction), and the influence of other wind directions is not considered here. Extending the previous model, a three-dimensional diffusion model with prevailing wind direction factors is built:

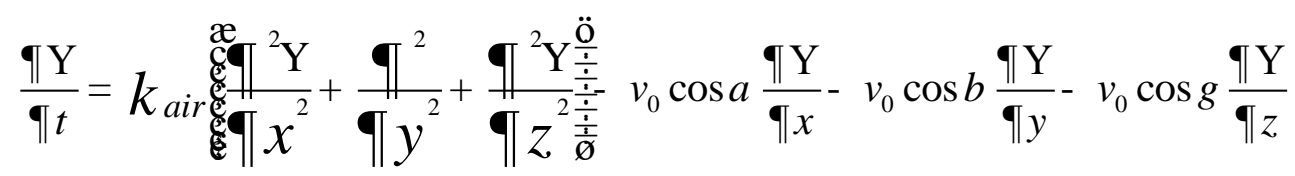

The average velocity of the prevailing wind direction is $v_{0}$, and the angles between the average velocity and the coordinate axis $x, y, z$ are respectively expressed as $\alpha, \beta, \gamma$.

Likewise, the heavy metal diffusion is regarded as the instantaneous and equal release of heavy metal pollutants to all directions from a certain pollution source. The initial condition is the point source function acting on the pollution source coordinate $\left(x_{0}, y_{0}, z_{0}\right)$, which can be recorded as:

$$
y\left(x_{0}, y_{0}, z_{0}\right)=M s\left(x_{0}, y_{0}, z_{0}\right)
$$

$M$ represents the total amount of heavy metal release, the initial concentration of heavy metals when time is 0 ; $s\left(x_{0}, y_{0}, z_{0}\right)$

is the point source function of the diffusion intensity in each unit. Then the solution of the equation (14) that satisfied the equation (15) is:

$$
\mathrm{Y}(x, y, z, t)=\frac{M}{\left(4 p k_{\text {air }} t\right)^{3 / 2}} e^{-\frac{\left(v_{0} \cos a x-x_{0}\right)^{2}+\left(v_{0} \cos b y-y_{0}\right)^{2}+\left(v_{0} \cos g z-z_{0}\right)^{2}}{{ }^{4} k_{a i r} t}}
$$

With the above results, the prevailing wind direction $v_{0}$, the angles $\alpha, \beta, \gamma$ between $v_{0}$ and the coordinate axis $x, y, z$, and other values such as $k_{\text {air }}$, the metal concentration at a given position is worked out.

The calculation results reveal that the existence of a certain prevailing wind direction will no longer lead to a homogeneous spherical diffusion of the heavy metals in the air. Instead, it will propagate in the certain direction of the wind. In addition, a lager wind speed will be followed by a higher proportion of heavy metals diffusion along the certain direction of the wind, which is complied with the actual situation, proving the accuracy of the model.

According to the above expansion of the two aspects of the model, human or natural factors such as wind direction, pollution control, precipitation, river trend, and soil composition may affect the propagation and diffusion of heavy metals. More detailed data will have a more optimized model.

\section{Conclusion}

In the paper, the Gaussian diffusion model and the two-dimensional diffusion model are applied to establish the propagation characteristics model of heavy metals in the atmosphere and soil moisture respectively, making the model more practical. Linear regression is a follow-up conduction to analyze the Gaussian diffusion model and twodimensional diffusion model. The location of the pollution source is scientifically determined by finding the extremum points and verifying them. Then, considering the prevailing wind direction, artificial control and other factors, a three-dimensional diffusion model is established and further optimized. The results obtained by the above models show that the factors affecting the analysis of the pollution source should be taken into consideration when determining the pollution source of heavy metals, and the pollution propagation mode should be analyzed as comprehensively as possible. When conducting heavy metal pollution control and treatment, a strong control on the pollution sources and the heavily polluted areas will gain a more significant effect. This paper is of certain referential significance for the study of determining heavy metal pollution sources. Through comprehensive consideration of factors and accurate data collection, the results will be more accurate and can lay a theoretical foundation for the treatment of heavy metal pollution.

However, due to the limited data obtained, the research in this paper still has some shortcomings, which are embodied in:

(1) The number of sampling points given in the question is small, which causes a large error after interpolation.

(2) The data provided in the question is sampled in a grid of one kilometer. No specific geographical distribution is given. Therefore, the analysis of pollution sources is based on interpolation, which is of certain ambiguity. 
(3) The paper lack of the data, the optimized model is not verified when introducing the model of the influence factors.

\section{References}

[1] Cheng Xiayan and Zhang Yun, 2012. "Journal of chongqing university of science and technology." Natural Science Edition,

[2] Zhao Jinjin. "Analytical method for heavy metal pollution sources in surface soils." Science \& Technology and Enterprise,

[3] Wang Ying, Jiang Xiaodong, Zhang Lu, and Liu Fei, 2014. "Analysis of heavy metal pollution sources in urban soil based on CDE." Technology Management Research,

[4] Zhang Ganlin, Zhu Yongguan, and Fu Bojie, 2003. "Evolution of urban soil quality and its ecoenvironmental effects." Acta Ecologica Sinica, vol. 23, pp. 539-546.

[5] Chen Ning, Chen Pingping, and Sun Shengli, 2011. "Diffusion model of space smoke cloud." Aerospace Electronic Warfare, pp. 23-25-49.

[6] Zhuo Jinwu, 2011. MATLAB application in mathematical modeling. Beijing: Beijing University of Aeronautics and Astronautics Press. pp. 180-181.

[7] Thomas, J., 1976. "A general gaussian diffusion-deposition model for elevated point sources." Journal of Applied Meteorology,

[8] Bilawal, R., Chongru, L., and Lili, W., 2017. "Least square method- a novel approach to determine symmetrical components of power system." Journal of Electrical Engineering \& Technology, vol. 12, pp. 39-44.

[9] Kevin Diamond, Thomas, F. J., and Michael, P. S., 2003. "Measurement of fluorophore concentrations and fluorescence quantum yield in tissue-simulating phantoms using three diffusion models of steady-state spatially." Physics in Medicine \& Biology,

[10] Nelson Fumo, M. A. and Rafe, B., 2015. "Regression analysis for prediction of residential energy consumption." Renewable and Sustainable Energy Reviews, vol. 47, pp. 332-343. 